\title{
TESTING ON NULL SEQUENCES IS ENOUGH FOR BOCHNER INTEGRABILITY
}

\author{
Fernando Mayoral and Pedro J. Paúl \\ Dedicated to Paul R. Halmos on the \\ occasion of his eightieth birthday.
}

Let $E$ be a normed space, a Fréchet space or a complete $(D F)$-space satisfying the dual density condition. Let $\Omega$ be a Radon measure space. We prove that a function $f: \Omega \rightarrow E$ is Bochner $p$-integrable if (and only if) $f$ is $p$-integrable with respect to the topology of uniform convergence on the norm-null sequences from $E^{\prime}$.

\section{INTRODUCTION}

Our first question was: Can one deduce that a function with values in a Banach space is Bochner integrable from the fact that it is integrable for a coarser topology? Of course the answer is negative for the weak topology (see [3, II.3.3 on p.53] for a concrete example or use the Dvoretzky-Rogers theorem in general). In this paper, we want to show that the answer is "Yes" for the topology of uniform convergence on the null sequences of the dual.

Let $\Omega$ be the measure space, $E$ be the Banach space and $\tau$ be the coarser topology. If $f: \Omega \rightarrow E$ is the $\tau$-integrable function candidate to be Bochner integrable, two problems are involved here: to prove that absolute integrability with respect to the $\tau$-seminorms implies that $t \rightarrow\|f(t)\|$ is in $L^{1}$ and to show that $f$ is norm-measurable if it is $\tau$-measurable. The aim of this paper is to show that there is a (somehow natural) class of spaces for which these two problems have a solution and that this class includes normed spaces, Fréchet spaces, strict $(L F)$-spaces and complete $(D F)$-spaces satisfying the dual density condition of Bierstedt and Bonet.

Received 8th November, 1995

This research has been supported by La Dirección General de Investigación Cientifica y Técnica, project PB94-1460, and by La Consejería de Educación y Ciencia de la Junta de Andalucía

Copyright Clearance Centre, Inc. Serial-fee code: 0004-9729/96 \$A2.00+0.00. 


\section{Terminology and Notation}

In what follows $(\Omega, \Sigma, \mu)$ stands for a $\sigma$-finite Radon measure space, where $\Omega$ is a locally compact and $\sigma$-compact topological space. Let $(E, \tau)$ be a locally convex space with a topology defined by a family of continuous seminorms $\mathcal{Q}(E, \tau)$. We consider measurability of functions in the sense of Lusin: we say that a function $f: \Omega \rightarrow E$ is $\tau$ measurable if there is a sequence $\left(K_{n}\right)$ (that we may take either disjoint or increasing) of compact sets such that the restriction $\left.f\right|_{K_{n}}$ is continuous for every $n \in \mathbb{N}$, and $\mu\left(\Omega \backslash \bigcup_{n} K_{n}\right)=0$. When $(E, \tau)$ is metrisable, the notion of a $\tau$-measurable function in the sense of Lusin coincides with the usual definition of a strongly measurable function as the $\mu$-almost everywhere limit of a sequence of simple functions. If $\tau_{1}$ and $\tau_{2}$ are two topologies defined on $E$, the identity $\left(E, \tau_{1}\right) \rightarrow\left(E, \tau_{2}\right)$ is said to be universally measurable if (among several equivalent conditions) every $\tau_{1}$-measurable function is also $\tau_{2}$-measurable (for arbitrary $\Omega$ and $\mu$ ).

A function $f: \Omega \rightarrow E$ is said to be integrable with respect to $\tau$, or simply $\tau$ integrable, if it is $\tau$-measurable and the scalar functions $q(f): t \in \Omega \rightarrow q(f(t)) \in \mathbb{R}$ are in $L^{1}(\mu)$ for every $q \in \mathcal{Q}(E, \tau)$. When $(E, \tau)$ is a Banach space, $\tau$-integrability equals Bochner integrability. $L^{1}(E, \tau)$ will denote the space of all (classes of $\mu$-almost everywhere equal) $\tau$-integrable functions endowed with the locally convex topology defined by the family of seminorms $f \rightarrow\|q(f)\|_{1}$ as $q \in \mathcal{Q}(E, \tau)$. For $1<p \leqslant \infty$, the space $L^{p}(E, \tau)$ is defined in the analogous way.

We say that a locally convex space $(E, \tau)$ has property $(B)$ of Pietsch if for each bounded subset $M$ of the space $\ell^{1}\{E, \tau\}$ of all absolutely summable sequences in $(E, \tau)$, there exists a disc $B \subset E$ such that for all $\left(x_{n}\right) \in M$ the following hold: $x_{n} \in E_{B}$ for each $n$ and $\sum_{n} p_{B}\left(x_{n}\right) \leqslant 1$, where $E_{B}$ is the linear span of $B$ and $p_{B}$ is its natural norm, the gauge of $B$. In other words, each bounded subset of $\ell^{1}\{E, \tau\}$ is a bounded subset of some $\ell^{1}\left\{E_{B}, p_{B}\right\}$. Metrisable and (df)-spaces have property $(B)$, for instance. A locally convex space $(E, \tau)$ is said to have property $(B M)$ if it has property $(B)$ and the topology $\tau$ is metrisable when restricted to bounded sets. Metrisable or, more generally, strict $(L F)$-spaces have property $(B M)$. For a quasi-complete locally convex space $(E, \tau)$ with property $(B M)$ the identity $\left(E, \sigma\left(E, E^{\prime}\right)\right) \rightarrow(E, \tau)$ is universally measurable $[4,4.13]$.

We introduced in $[4,3.5]$ the notion of fundamental $L^{p}$-boundedness as an extension of property $(B)$. Let $1 \leqslant p \leqslant \infty$. A locally convex space $(E, \tau)$ is said to be fundamentally $L^{p}$-bounded, with respect to $(\Omega, \Sigma, \mu)$, if each bounded subset $M$ of $L^{p}\{E, \tau\}$ is contained in a bounded set of the form

$$
\left[U_{p}, B\right]:=\left\{f \in L^{p}\{E, \tau\}: f(t) \in E_{B} \text { almost everywhere and } p_{B}(f) \in U_{p}\right\},
$$

where $B$ is a disc in $E$ and $U_{p}$ stands for the unit ball of $L^{P}(\mu)$. This definition 
applied to the particular case of the counting measure on the power set of $\mathbb{N}$, tells us that fundamental $\ell^{1}$-boundedness is just property $(B)$ (this is the terminology of $[7]$, by the way).

The dual density condition was introduced by Bierstedt and Bonet in connection with their solution to the problem of when a Köthe echelon space is distinguished. They proved [1, Theorem 5] that a $(D F)$-space $E$ satisfies the dual density condition if and only if every bounded subset of $E$ is metrisable, or if and only if $\ell^{\infty}(E, \tau)$ is quasi-barrelled. $(D F)$-spaces satisfying the dual density condition are quasi-barrelled (but not the opposite!). In particular, for $(D F)$-spaces property $(B M)$ equals the dual density condition.

We refer the reader to the books by Jarchow [5], Köthe [6], Pérez Carreras and Bonet [7] or Robertson and Robertson [8] for the terminology about locally convex spaces and to the monographs by Bourbaki [2], Diestel and Uhl [3], Schwartz [9] or Thomas [10] for the properties of measurable functions. Our paper [4] contains several results about localisation of bounded sets in $L^{p}(E, \tau)$ and Radon-Nikodym theorems.

\section{REsults}

MAIN TheOREM. Let $(E, \tau)$ be a locally convex space. Let $\tau_{0}$ be another locally convex topology on $E$ coarser that $\tau$ and such that

(1) the identity $\left(E, \tau_{0}\right) \rightarrow(E, \tau)$ is universally measurable,

(2) every $\tau_{0}$-bounded subset of $E$ is also $\tau$-bounded,

(3) the space $\left(E, \tau_{0}\right)$ is fundamentally $L^{p}$-bounded for some $p \in[1, \infty)$.

Then a function $f: \Omega \rightarrow E$ is $p$-integrable with respect to $\tau$ if (and only if) $f$ is $p$-integrable with respect to $\tau_{0}$, that is

$$
L^{p}(E, \tau)=L^{p}\left(E, \tau_{0}\right)
$$

holds as an equality of vector spaces.

Proof: Let $f: \Omega \rightarrow E$ be $\tau_{0}$-integrable. Since the identity $\left(E, \tau_{0}\right) \rightarrow(E, \tau)$ is universally measurable, it follows that the function $f$ is $\tau$-measurable. It remains to prove that for every $\tau$-continuous seminorm $q$ the scalar function $t \rightarrow q(f(t))$ is in $L^{p}(\mu)$. The space $\left(E, \tau_{0}\right)$ is fundamentally $L^{p}$-bounded, therefore there exists a disc $B$ in $\left(E, \tau_{0}\right)$ such that the scalar function $t \rightarrow p_{B}(f(t))$ is in $L^{p}(\mu)$. Since $B$ is also $\tau$-bounded, it is contained in some multiple of the unit ball of $q$, hence $t \rightarrow q(f(t))$ is in $L^{p}(\mu)$, as desired.

We shall give several applications of this theorem.

COROLlaRY 1. Let $E$ be a normed space and let $\tau_{0}$ be the topology of uniform convergence on the sequences that converge to zero in $E^{\prime}$. Let $p \in[1, \infty)$. Then a function $f: \Omega \rightarrow E$ is Bochner $p$-integrable if (and only if) $f \in L^{p}\left(E, \tau_{0}\right)$. 
Proof: We only have to check that conditions (1) and (3) above hold in this case. A consequence of the Grothendieck-Phillips theorem [9, Part II, I.1. Theorem 3 on p.162] or $\left[10\right.$, p.50], is that for a Banach space $E$ the identity $\left(E, \sigma\left(E, E^{\prime}\right)\right) \rightarrow(E,\|\cdot\|)$ is universally measurable. It is easy to see, by passing to its completion, that the same holds when $E$ is a non-complete normed space. This proves (1). To see (3) note that $\left(E, \tau_{0}\right)$ is a $(d f)$-space $[5,12.4-5]$, that is, it has a fundamental sequence of bounded sets (the integer multiples of the unit ball) and every norm-null sequence in $E^{\prime}$ is equicontinuous. Now $[4,3.10]$ states that $(d f)$-spaces are fundamentally $L^{p}$-bounded for every $p \in[1, \infty)$.

Remarks. Given $p \in[1, \infty)$, Corollary 1 tells us, in other words, that if $E$ is a normed space and $f: \Omega \rightarrow E$ is strongly measurable then $f$ is Bochner $p$-integrable provided that for every null sequence $\left(x_{n}^{\prime}\right)$ from $E^{\prime}$, the scalar function

$$
t \in \Omega \rightarrow \sup \left\{\left|\left\langle f(t), x_{n}^{\prime}\right\rangle\right|: n \in \mathbb{N}\right\}
$$

is $p$-integrable.

The $(d f)$-space $\left(E, \tau_{0}\right)$ is not complete if $E$ is not reflexive $[5,12.5 .1$ and 2$]$. If $A$ is a measurable set, the integral $\int_{A} f d \mu$ of a function $f \in L^{1}\left(E, \tau_{0}\right)$ is obtained as the limit of a net of Riemann sums so that they belong, a priori, to the completion of $\left(E, \tau_{0}\right)$ and this completion coincides (as a vector space) with the bidual $E^{\prime \prime}[5,12.5 .1]$. However, it follows from Corollary 1 that these integrals are, indeed, elements of $E$.

Corollary 1 also holds for every locally convex topology between $\tau_{0}$ and the norm topology. For all of these topologies $E$ is again a ( $d f$ )-space.

Let us consider now the situation on the dual $E^{\prime}$ of a Banach space $E$. The difficulty is to lift integrability from the topology $\tau_{0}^{\prime}$ of uniform convergence on the null sequences on $E$ to the norm topology in $E^{\prime}$. The main problem will be that the behaviour of the measurability is not so good; a measurable function with respect the weak *-topology $\sigma\left(E^{\prime}, E\right)$ may not be measurable with respect the norm topology on $E^{\prime}$ as the cases [9, Exercise 1 and 2 on p.168] $E=\ell^{1}$ or $E=C[0,1]$ show.

Corollary 2. Let $E$ be a Banach space with dual $E^{\prime}$ and $p \in[1, \infty)$. Then the following hold.

(a) A strongly measurable function $f: \Omega \rightarrow E^{\prime}$ is Bochner p-integrable if and only if for every null sequence $\left(x_{n}\right)$ in $E$ the scalar function

$$
t \in \Omega \rightarrow \sup \left\{\left|\left\langle x_{n}, f(t)\right\rangle\right|: n \in \mathbb{N}\right\}
$$

is in $L^{p}(\mu)$.

(b) If $E^{\prime}$ is separable, then a function $f: \Omega \rightarrow E^{\prime}$ is Bochner p-integrable if and only if it is p-integrable with respect to the topology $\tau_{0}^{\prime}$ of uniform convergence on the norm-null sequences in $E$. 
Proof: Part (a) can be proved as in the Main Theorem using the fact that $\left(E^{\prime}, \tau_{0}^{\prime}\right)$ is also a $(d f)$-space and so is fundamentally $L^{p}$-bounded. Part (b) follows from a theorem due to Meyer and Schwartz [9, Part I, II.3 Corollary 2 of Theorem 10 on pp.122$124]\left[10\right.$, p.51] stating that if $E^{\prime}$ is separable then the identity $\left(E^{\prime}, \sigma\left(E^{\prime}, E\right)\right) \rightarrow\left(E^{\prime},\|\cdot\|\right)$ is universally measurable.

By the Banach-Dieudonné theorem, $\tau_{0}^{\prime}$ equals the topology of uniform convergence on the compact subsets of $E[\mathbf{5}, 9.4 .3]$. Moreover, $\left(E^{\prime}, \tau_{0}^{\prime}\right)$ is not only a (df)-space; it is also a complete, Schwartz $(g D F)$-space $[5,9.4 .1-3,11.1 .4$ and 12.5.2 and 6].

Corollary 3. Let $p \in[1, \infty)$ and $(E, \tau)$ be a complete $(D F)$-space with the dual density condition. Let $\tau_{0}$ be the topology of uniform convergence on the sequences from $E^{\prime}$ that converge to zero in the strong topology $\beta\left(E^{\prime}, E\right)$. Then a function $f$ : $\Omega \rightarrow E$ is $p$-integrable with respect to $\tau$ if (and only if) $f$ is p-integrable with respect to $\tau_{0}$.

Proof: Since every $(D F)$-space with the dual density condition has property $(B M)$, condition (1) is a particular case of $[4,4.13]$. On the other hand, it is clear that if $(E, \tau)$ is a $(D F)$-space then $\left(E, \tau_{0}\right)$ is a $(d f)$-space and the proof finishes as in the proof of Corollary 1.

Corollary 3 can be also obtained as a consequence of Corollary 4 below - the corresponding result for quasi-complete spaces having property $(B M)-$ but the proof of the latter requires more work.

LEMMA . Let $(E, \tau)$ be a quasi-complete locally convex space with property $(B M)$ and let $\tau_{0}$ be the topology of uniform convergence on the sequences that converge to zero in $\left(E^{\prime}, \beta\left(E^{\prime}, E\right)\right)$. Then $\left(E, \tau_{0}\right)$ is fundamentally $L^{p}$-bounded for each $p \in[1, \infty)$.

Proof: We start by proving that $\left(E, \tau_{0}\right)$ has property $(B)$. Since $(E, \tau)$ has property $(B)$, it will be enough to prove that if $M$ is a bounded subset of $\ell^{1}\left\{E, \tau_{0}\right\}$ then $M$ is also bounded in $\ell^{1}\{E, \tau\}$. Let $q$ be any $\tau$-continuous seminorm and assume that

$$
\sup \left\{\sum_{n=1}^{\infty} q\left(x_{n}\right):\left(x_{n}\right) \in M\right\}=\infty .
$$

Then there exists a sequence $\left\{\left(x_{n}^{(k)}\right)_{n}: k=1,2, \ldots\right\} \subset M$ and an increasing sequence of indices $\left(n_{k}\right)$ such that

$$
\sum_{n=1+n_{k}}^{n_{k+1}} q\left(x_{n}^{(k)}\right)>2^{2 k} .
$$

Let $V \subset E^{\prime}$ be the polar of the unit ball associated to $q$. Then we can find a sequence 
$\left(v_{n}\right) \subset V$ such that

$$
\sum_{n=1+n_{k}}^{n_{k+1}}\left\langle x_{n}^{(k)}, v_{n}\right\rangle>2^{2 k} \quad \text { for every } k=1,2, \ldots
$$

Since $V$ is $\beta\left(E^{\prime}, E\right)$-bounded it follows that the sequence

$$
\frac{v_{1}}{2^{0}}, \ldots, \frac{v_{n_{1}}}{2^{0}}, \frac{v_{1+n_{1}}}{2^{1}}, \ldots, \frac{v_{n_{2}}}{2^{1}}, \frac{v_{1+n_{2}}}{2^{2}}, \ldots, \frac{v_{n_{3}}}{2^{2}}, \ldots
$$

converges to zero in the strong topology $\beta\left(E^{\prime}, E\right)$ and satisfies

$$
\sum_{n=1+n_{k}}^{n_{k+1}}\left\langle x_{n}^{(k)}, \frac{v_{n}}{2^{k}}\right\rangle>2^{k} \quad \text { for every } k=1,2, \ldots,
$$

contradicting the fact that $M$ is bounded in $\ell^{1}\left\{E, \tau_{0}\right\}$.

We now prove that $\left(E, \tau_{0}\right)$ is fundamentally $L^{1}(\mu)$-bounded. By $[4,4.13]$ the identity $\left(E, \sigma\left(E, E^{\prime}\right)\right) \rightarrow(E, \tau)$ is universally measurable so that the identity $\left(E, \tau_{0}\right) \rightarrow$ $(E, \tau)$ will also be universally measurable. (This proves, by the way, that condition (1) of the Main Theorem is satisfied.) Therefore, given $f \in L^{1}\left(E, \tau_{0}\right)$ there is a disjoint sequence $\left(K_{n}\right)$ of compact sets such that the restriction $\left.f\right|_{K_{n}}$ is $\tau$-continuous for every $n \in \mathbb{N}$, and $\mu\left(\Omega \backslash \cup_{n} K_{n}\right)=0$. In particular (see [2]), $f$ will be integrable on every measurable set $A$ contained in some $K_{n}$; that is, there is an element $\int_{A} f d \mu \in E$ such that

$$
\left\langle\int_{A} f d \mu, v\right\rangle=\int_{A}\langle f(t), v\rangle d \mu \quad \text { for every } v \in E^{\prime} .
$$

Let $F \subset L^{1}\left(E, \tau_{0}\right)$ be a bounded set. For each $q \in \mathcal{Q}\left(E, \tau_{0}\right)$ take

$$
\rho_{q}:=\sup \left\{\int_{\Omega} q(f) d \mu: f \in F\right\}<\infty .
$$

Let $F_{0}$ be the set of all $E$-valued sequences of the form

$$
\left(\int_{A_{1}} f d \mu, \int_{A_{2}} f d \mu, \ldots\right)
$$

where $f \in F$ and $A_{1}, A_{2}, \ldots$ is a sequence of pairwise disjoint, measurable sets with positive and finite measure such that each one of them is contained in some compact set where $f$ is $r$-continuous. As pointed out above, for each $f \in F$ there is at least one such sequence $\left(A_{n}\right)$. For each seminorm $q \in \mathcal{Q}(E, \tau)$ and each of these sequences, we have

$$
\sum_{n=1}^{\infty} q\left(\int_{A_{n}} f d \mu\right) \leqslant \sum_{n=1}^{\infty} \int_{A_{n}} q(f) d \mu \leqslant \int_{\Omega} q(f) d \mu \leqslant \rho_{q} .
$$


This tells us that $F_{0}$ is a bounded subset of $\ell^{1}\left\{E, \tau_{0}\right\}$. We have proved above that $\left(E, \tau_{0}\right)$ has property $(B)$, hence there is a closed disc $B \subset E$ such that for every sequence $\left(x_{n}\right) \in F_{0}$ we have $x_{n} \in E_{B}$ for each $n \in \mathbb{N}$, and $\sum_{n=1}^{\infty} p_{B}\left(x_{n}\right) \leqslant 1$. We shall prove that for every $f \in F$ we have (i) $f(t) \in E_{B}$ almost everywhere and (ii) the function $t \rightarrow p_{B}(f(t))$ is in the unit ball of $L^{1}(\mu)$.

(i) If there is $f \in F$ such that $\mu\left\{t \in \Omega: f(t) \notin E_{B}\right\}>0$, then there is a compact set $K \subset \Omega$ with positive measure such that $f: K \rightarrow E$ is $\tau$-continuous and $f(t) \notin E_{B}$ for all $t \in K$. By $[4,3.7]$ for every $n \in \mathbb{N}$ there exists a simple function $z_{n}: K \rightarrow B^{\circ} \subset$ $E^{\prime}$ such that $\operatorname{Re}\left\langle f(t), z_{n}(t)\right\rangle>n$ for all $t \in K$. If we write $z_{n}=\sum_{i=1}^{k} v_{i} \chi_{A_{i}}$, where $\left\{A_{1}, A_{2}, \ldots, A_{k}\right\}$ is a measurable partition of $K$ and $\left\{v_{1}, v_{2}, \ldots, v_{k}\right\} \subset B^{\circ}$, then the sequence

$$
\left(\int_{A_{1}} f d \mu, \int_{A_{2}} f d \mu, \ldots, \int_{A_{k}} f d \mu, 0,0, \ldots\right)
$$

is in $F_{0}$. However, we also have

$$
\begin{aligned}
\sum_{i=1}^{k} p_{B}\left(\int_{A_{i}} f d \mu\right) & \geqslant \sum_{i=1}^{k}\left|\operatorname{Re}\left\langle\int_{A_{i}} f d \mu, v_{i}\right\rangle\right|=\sum_{i=1}^{k}\left|\int_{A_{i}} \operatorname{Re}\left\langle f, v_{i}\right\rangle d \mu\right| \\
& \geqslant n \sum_{i=1}^{k} \mu\left(A_{i}\right)=n \mu(K),
\end{aligned}
$$

contradicting the boundedness of $F_{0}$ in $\ell^{1}\left\{E_{B}, p_{B}\right\}$.

(ii) Assume that the set functions $\left\{p_{B}(f): f \in F\right\}$ is not contained in the unit ball of $L^{1}(\mu)$. This can happen because this set is not contained in $L^{1}(\mu)$ at all, or simply because $\left\|p_{B}(f)\right\|_{1}>1$ for some $f \in F$. In either case, we can find a function $f \in F$ and a compact set $K \subset \Omega$, such that the functions $f: K \rightarrow E$ and $p_{B}(f): K \rightarrow \mathbb{R}$ are $\tau$-continuous, and $\left\|p_{B}(f) \cdot \chi_{K}\right\|_{1}>1+\delta$, for some positive $\delta$. It is well-known that for $\varphi \in L^{1}(\mu)$ one has

$$
\|\varphi\|_{1}=\sup \left\{\left|\int_{\Omega} \varphi \cdot \theta d \mu\right|: \theta \text { a simple function with }\|\theta\|_{\infty} \leqslant 1\right\},
$$

so we can find a simple function $\theta$ in the unit ball of $L^{\infty}(\mu)$ such that $\int_{K} p_{B}(f) \cdot \theta d \mu>$ $1+\delta$; note that we may assume that $\theta$ is non-negative. Again by [4, 3.7], given $\varepsilon>0$ there is a simple function $z: K \rightarrow B^{\circ} \subset E^{\prime}$ such that

$$
p_{B}(f(t))<\operatorname{Re}\langle f(t), z(t)\rangle+\varepsilon \quad \text { for all } t \in K .
$$

Write $\theta$ and $z$ as

$$
\theta=\sum_{i=1}^{k} \alpha_{i} \chi_{A_{i}} \quad z=\sum_{i=1}^{k} v_{i} \chi_{A_{i}}
$$


where the sets $\left(A_{i}\right)$ are pairwise disjoint and have positive finite measure, and each $\alpha_{i}$ is in $[0,1]$. Take the sequence $\left(x_{i}\right) \subset E$ defined by $x_{i}:=\int_{A_{i}} f d \mu$, for $i=1,2, \ldots, k$ and $x_{i}=0$ afterwards. Then $\left(x_{i}\right)$ is in $F_{0}$ because each $A_{i}$ is contained in $K$, where $f$ is $\tau$-continuous. Now, since each $\alpha_{i}$ is in $[0,1]$ and $F_{0}$ is contained in the unit ball of $\ell^{1}\left\{E_{B}, p_{B}\right\}$, we have that

$$
\begin{aligned}
1 & \geqslant \sum_{i=1}^{k} \alpha_{i} p_{B}\left(x_{i}\right)=\sum_{i=1}^{k} \alpha_{i} p_{B}\left(\int_{A_{i}} f d \mu\right) \geqslant \sum_{i=1}^{k} \alpha_{i} \operatorname{Re}\left\langle\int_{A_{i}} f d \mu, v_{i}\right\rangle \\
& =\sum_{i=1}^{k} \alpha_{i} \int_{A_{i}} \operatorname{Re}\left\langle f, v_{i}\right\rangle d \mu \geqslant \sum_{i=1}^{k} \alpha_{i} \int_{A_{i}}\left(p_{B}(f)-\varepsilon\right) d \mu \\
& =\int_{K} \theta p_{B}(f) d \mu-\varepsilon\left\|\theta \chi_{K}\right\|_{1}>1+\delta-\varepsilon \mu(K),
\end{aligned}
$$

where the last inequality holds because $\int_{K} p_{B}(f) \cdot \theta d \mu>1+\delta$, on the one hand, and $\left\|\theta \chi_{K}\right\|_{1} \leqslant\|\theta\|_{\infty}\left\|\chi_{K}\right\|_{1} \leqslant \mu(K)$, on the other. Since $\varepsilon$ was arbitrary, we obtain a contradiction.

To finish the proof of the Lemma apply $[4,3.6]$; this result tells us that fundamental $L^{1}(\mu)$-boundedness implies fundamental $L^{p}$-boundedness for every $p \in[1, \infty]$.

Corollary 4. Let $p \in[1, \infty)$ and let $(E, \tau)$ be a Fréchet space or, more generally, a strict $(L F)$-space. Let $\tau_{0}$ be the topology of uniform convergence on the sequences from $E^{\prime}$ that converge to zero in the strong topology $\beta\left(E^{\prime}, E\right)$. Then a function $f: \Omega \rightarrow E$ is p-integrable with respect to $\tau$ if (and only if) $f$ is p-integrable with respect to $\tau_{0}$.

\section{REFERENCES}

[1] K.D. Bierstedt and J. Bonet, 'Dual density conditions in (DF)-spaces. I', Results Math. 14 (1988), 242-274.

[2] N. Bourbaki, Intégration (Hermann, Paris, 1973).

[3] J. Diestel and J.J. Uhl, Vector measures (American Mathematical Society, Providence, R.I., 1977).

[4] M. Florencio, F. Mayoral and P.J. Paúl, 'Spaces of vector-valued integrable functions and localization of bounded subsets', Math. Nachr. 174 (1995), 89-111.

[5] H. Jarchow, Locally convex spaces (B.G. Teubner, Stuttgart, 1981).

[6] G. Köthe, Topological vector spaces. I, II (Springer-Verlag, Berlin, Heidelberg, New York, 1969, 1979).

[7] P. Pérez Carreras and J. Bonet, Barrelled locally convex spaces (North-Holland, Amsterdam, New York, Oxford, Tokyo, 1987).

[8] A.P. Robertson and W.J. Robertson, Topological vector spaces, (second edition) (Oxford University Press, London, 1973). 
[9] L. Schwartz, Radon measures on arbitrary topological spaces and cylindrical measures, Tata Institute of Fundamental Research Studies (North-Holland, Amsterdam, New York, Oxford, Tokyo, 1987).

[10] G.E.F. Thomas, 'The Lebesgue-Nikodym theorem for vector valued Radon measures', Mem. Amer. Math. Soc. 139 (1974).

E.S. Ingenieros Industriales

Avda. Reina Mercedes s/n

41012-Sevilla

Spain

email: PITI@CICA.ES 